\title{
Erratum to: Burniat surfaces II: secondary Burniat surfaces form three connected components of the moduli space
}

\author{
I. Bauer • F. Catanese
}

Published online: 3 June 2014

(C) Springer-Verlag Berlin Heidelberg 2014

\section{Erratum to: Invent Math (2010) 180:559-588 \\ DOI 10.1007/s00222-010-0237-z}

The main goal of this note is to correct an erroneous statement in the originally published article: nodal Burniat surfaces with $K_{S}^{2}=4$ do not form a connected component of the moduli space, but are properly contained in a unique irreducible connected component. Therefore the title should be amended to 'Secondary Burniat surfaces yield three connected components of the moduli space'.

Theorem 1.1. in the originally published article is incorrect. It has to be replaced by the following statement:

Theorem 1.1 The subset of the moduli space of canonical surfaces of general type $\mathfrak{M}_{1,4}^{c a n}$ given by the union of the open set corresponding to extended Burniat surfaces with $K_{S}^{2}=4$ with the irreducible closed set parametrizing nodal Burniat surfaces with $K_{S}^{2}=4$ is an irreducible connected component, normal, unirational of dimension 3.

The online version of the original article can be found under doi:10.1007/s00222-010-0237-z.

\footnotetext{
I. Bauer · F. Catanese $(\varangle)$

Lehrstuhl Mathematik VIII, Mathematisches Institut der Universität Bayreuth NW II, Universitätsstr. 30, 95447 Bayreuth, Germany

e-mail: fabrizio.catanese@uni-bayreuth.de

I. Bauer

e-mail: ingrid.bauer@uni-bayreuth.de
} 
Moreover the base of the Kuranishi family of deformations of such a minimal model $S$ is smooth.

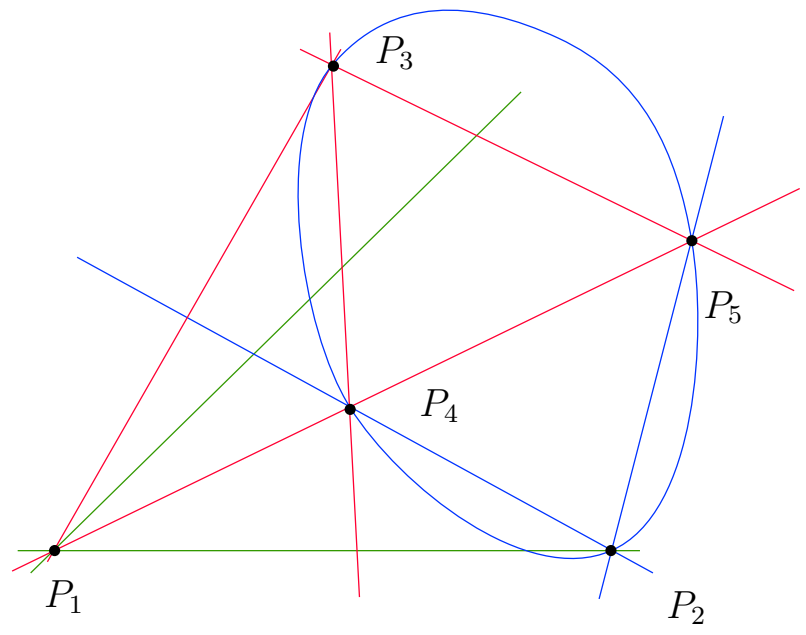

Details and proofs of the amended statements can be found in [1], in particular the complicated definition of extended Burniat surfaces. It may suffice here to say that these are birational to bidouble covers (i.e., Galois covers with group $(\mathbb{Z} / 2)^{2}$ ) of the plane with branch divisors consisting of lines and one conic as in the picture above.

First of all, let's point out the main philosophical mistake we made.

Let $S$ be a minimal surface of general type and let $X$ be its canonical model. Denote by $\operatorname{Def}(S)$, resp. $\operatorname{Def}(X)$, the base of the Kuranishi family of $S$, resp. of $X$. Assume also that we have a group of automorphisms $1 \neq G \leq \operatorname{Aut}(S)=$ $\operatorname{Aut}(X)$.

Then we can consider the Kuranishi space of $G$-invariant deformations of $S$, denoted by $\operatorname{Def}(S, G)$, and respectively consider $\operatorname{Def}(X, G)$; we have then a natural map $\operatorname{Def}(S, G) \rightarrow \operatorname{Def}(X, G)$.

We asserted (in the originally published article, page 577 , sentence after the proof of proposition 2.19) that since all the deformations of the canonical model $X$ preserve the group action, then the same must hold for the deformations of the minimal model $S$ : in other words, that the above natural map is surjective.

This is not true, not only in general, but exactly in this special case (see [1], and also [3] for more details).

Theorem 1.2 The deformations of nodal Burniat surfaces with $K_{S}^{2}=4$ to extended Burniat surfaces with $K_{S}^{2}=4$ yield examples where $\operatorname{Def}\left(S,(\mathbb{Z} / 2 \mathbb{Z})^{2}\right)$ $\rightarrow \operatorname{Def}\left(X,(\mathbb{Z} / 2 \mathbb{Z})^{2}\right)$ is not surjective.

Moreover, whereas for the canonical model we have:

$$
\operatorname{Def}\left(X,(\mathbb{Z} / 2 \mathbb{Z})^{2}\right)=\operatorname{Def}(X),
$$


for the minimal models

$\operatorname{Def}\left(S,(\mathbb{Z} / 2 \mathbb{Z})^{2}\right) \subsetneq \operatorname{Def}(S)$,

and the subset $\operatorname{Def}\left(S,(\mathbb{Z} / 2 \mathbb{Z})^{2}\right)$ corresponds to the locus of nodal Burniat surfaces.

The incorrect statement of Theorem 1.1. in the originally published article stems also from a technical error in Lemma 2.10 affecting Corollary 2.11; in particular, even if the calculation of the dimension of $H^{1}\left(\Theta_{S}\right)$ was correct, the determination of the eigenspaces for the $(\mathbb{Z} / 2 \mathbb{Z})^{2}$-action was incorrect.

Parts (1), (2) and (3) of the following lemma (Proposition 7.1 of [1]) were contained in Lemma 2.10 of the originally published article, while (4) corrects a wrongly stated assertion of (2) of loc. cit.

Lemma 1.3 Consider a finite set of distinct linear forms

$$
l_{\alpha}:=y-c_{\alpha} x, \quad \alpha \in A
$$

vanishing at the origin in $\mathbb{C}^{2}$.

Let $p: Z \rightarrow \mathbb{C}^{2}$ be the blow up of the origin, let $D_{\alpha}$ be the strict transform of the line $L_{\alpha}:=\left\{l_{\alpha}=0\right\}$, and let $E$ be the exceptional divisor.

Let $\Omega_{\mathbb{C}^{2}}^{1}\left(\left(\mathrm{~d} \log l_{\alpha}\right)_{\alpha \in A}\right)$ be the sheaf of rational 1-forms $\eta$ generated by $\Omega_{\mathbb{C}^{2}}^{1}$ and by the differential forms $d \log l_{\alpha}$ as an $\mathcal{O}_{\mathbb{C}^{2} \text {-module and define similarly }}$ $\Omega_{Z}^{1}\left(\left(\log D_{\alpha}\right)_{\alpha \in A}\right)$. Then:

(1) $p_{*}\left(\Omega_{Z}^{1}(\log E)(-E)\right)=\Omega_{\mathbb{C}^{2}}^{1}$,

(2) $p_{*}\left(\Omega_{Z}^{1}\left(\log E,\left(\log D_{\alpha}\right)_{\alpha \in A}\right)\right)=\Omega_{\mathbb{C}^{2}}^{1}\left(\left(\operatorname{dlog} l_{\alpha}\right)_{\alpha \in A}\right)$,

(3) $p_{*}\left(\Omega_{Z}^{1}\left(\left(\log D_{\alpha}\right)_{\alpha \in A}\right)\right)=\left\{\eta \in \Omega_{\mathbb{C}^{2}}^{1}\left(\left(\operatorname{dlog} l_{\alpha}\right)_{\alpha \in A}\right) \mid\right.$

$\left.\eta=\sum_{\alpha} g_{\alpha} \mathrm{d} \log l_{\alpha}+\omega, \omega \in \Omega_{\mathbb{C}^{2}}^{1}, \sum_{\alpha} g_{\alpha}(0)=0\right\}$.

(4) $p_{*}\left(\Omega_{Z}^{1}\left(\left(\log D_{\alpha}\right)_{\alpha \in A}\right)(E)\right) \supset \Omega_{\mathbb{C}^{2}}^{1}\left(\left(\operatorname{dlog} l_{\alpha}\right)_{\alpha \in A}\right)$ and

$$
\operatorname{dim}_{\mathbb{C}}\left[p_{*}\left(\Omega_{Z}^{1}\left(\left(\log D_{\alpha}\right)_{\alpha \in A}\right)(E)\right) / \Omega_{\mathbb{C}^{2}}^{1}\left(\left(\operatorname{dlog} l_{\alpha}\right)_{\alpha \in A}\right)\right]=d-2
$$

is supported at the origin, where $d:=|A|$. More precisely, we have an exact sequence

$$
0 \rightarrow \Omega_{\mathbb{C}^{2}}^{1} \rightarrow p_{*}\left(\Omega_{Z}^{1}\left(\left(\log D_{\alpha}\right)_{\alpha \in A}\right)(E)\right) \rightarrow \bigoplus_{\alpha=1}^{d} \mathcal{O}_{D_{\alpha}}(0) \rightarrow \mathbb{C}_{0}^{2} \rightarrow 0
$$

(5) Assume w.l.o.g. $c_{1}=0$ in the following formulae: then $p_{*}\left(\Omega_{Z}^{1}\left(\log D_{1}\right)\right.$ $(-E)) \subset \Omega_{\mathbb{C}^{2}}^{1}\left(\operatorname{dlog} l_{1}\right)$ is the subsheaf of forms 


$$
\left\{\omega=\alpha d x+\beta \frac{d y}{y} \mid \beta(0)=0, \frac{\partial \beta}{\partial y}(0)=0, \frac{\partial \beta}{\partial x}(0)+\alpha(0)=0\right\} .
$$

(6) $p_{*}\left(\Omega_{Z}^{1}(-E)\right)=\mathfrak{m}_{0} \Omega_{\mathbb{C}^{2}}^{1}$.

(7) $p_{*}\left(\Omega_{Z}^{1}\left(\log D_{1}, \log D_{2}\right)(-E)\right) \subset \Omega_{\mathbb{C}^{2}}^{1}\left(\mathrm{~d} \log l_{1}\right.$, d log $\left.l_{2}\right)$ is the subsheaf of forms

$$
\left\{\omega=\alpha \frac{d x}{x}+\beta \frac{d y}{y} \mid \alpha(0)=0, \beta(0)=0, \frac{\partial(\alpha+\beta)}{\partial x}(0)=0, \frac{\partial(\alpha+\beta)}{\partial y}(0)=0\right\} .
$$

As a consequence Corollary 2.11 of the originally published article has also to be corrected for nodal Burniat surfaces with $K_{S}^{2}=4$ in the following way (see table 1 page 1112 of [1]):

Corollary 1.4 (1) Let $S$ be the minimal model of a nodal Burniat surface with $K_{S}^{2}=4$.

Then the dimensions of the eigenspaces of the cohomology groups of the tangent sheaf $\Theta_{S}$ (for the natural $(\mathbb{Z} / 2 \mathbb{Z})^{2}$-action) are as follows.

- $h^{1}\left(S, \Theta_{S}\right)^{\mathrm{inv}}=2, h^{2}\left(S, \Theta_{S}\right)^{\mathrm{inv}}=0$,

- $h^{1}\left(S, \Theta_{S}\right)^{3}=1=h^{2}\left(S, \Theta_{S}\right)^{3}$,

- $h^{j}\left(S, \Theta_{S}\right)^{i}=0$, for $i \in\{1,2\}$.

(2) Let $S$ be the minimal model of an extended Burniat surface with $K_{S}^{2}=4$. Then the dimensions of the eigenspaces of the cohomology groups of the tangent sheaf $\Theta_{S}$ (for the natural $(\mathbb{Z} / 2 \mathbb{Z})^{2}$-action) are as follows.

- $h^{1}\left(S, \Theta_{S}\right)^{\text {inv }}=3, h^{2}\left(S, \Theta_{S}\right)^{\text {inv }}=0$,

- $h^{1}\left(S, \Theta_{S}\right)^{i}=0=h^{2}\left(S, \Theta_{S}\right)^{i}$, for $i \in\{1,2\}$,

- $h^{1}\left(S, \Theta_{S}\right)^{3}=0, h^{2}\left(S, \Theta_{S}\right)^{3}=1$.

For details of the proof of Theorem 1.1 we refer to [1], Theorem 5.1.

\section{References}

1. Bauer, I., Catanese, F.: Burniat surfaces III: deformations of automorphisms and extended Burniat surfaces. Documenta Mathematica 18, 1089-1136 (2013)

2. Burns Jr., D.M., Wahl, J.: Local contributions to global deformations of surfaces. Invent. Math. 26, 67-88 (1974)

3. Catanese, F.: A superficial working guide to deformations and moduli. In: Handbook of Moduli, in Honour of David Mumford. Advanced Lectures in Mathematics 26, vol. I, pp. 161-216. International Press and Higher Education Press, Boston (2013)

4. Vakil, R.: Murphy's law in algebraic geometry: badly-behaved deformation spaces. Invent. Math. 164(3), 569-590 (2006) 